PROCEEDINGS OF THE

AMERICAN MATHEMATICAL SOCIETY

Volume 126, Number 4, April 1998, Pages 1081-1088

S 0002-9939(98)04094-5

\title{
COMPACT QUANTUM GROUPS ASSOCIATED WITH MONOIDAL FUNCTORS
}

\author{
HUU HUNG BUI
}

(Communicated by Palle E. T. Jorgensen)

\begin{abstract}
We provide a $C^{*}$-algebra structure on the bialgebra associated with a monoidal linear ${ }^{*}$-functor. The $C^{*}$-algebra obtained in this way is a compact quantum group in the sense of Baaj and Skandalis. We show that the category of finite dimensional unitary corepresentations of this $C^{*}$-algebra is equivalent to the given category.
\end{abstract}

\section{INTRODUCTION}

Woronowicz ([W]) and Baaj and Skandalis ([BS] and [Sk]) defined compact quantum groups as the $C^{*}$-algebras generated by the matrix elements of their corepresentations. On the other hand, monoidal categories now form the right framework for the study of quantum groups; see [JS], [JS2], [K], [KT] and [Y]. In [JS], Joyal and Street constructed a bialgebra $\operatorname{End}^{\vee}(X)$ from a given monoidal functor $X$ satisfying suitable conditions. This bialgebra plays an important role in the modern treatment of Tannaka reconstruction; see [JS], [Sc] and [D].

In this paper we establish the relationship between these two methods. We provide a $C^{*}$-algebra structure on $\operatorname{End}^{\vee}(X)$, and then show that the completion of End $^{\vee}(X)$ is a compact quantum group in the Baaj-Skandalis sense. We also show that the category of finite dimensional unitary corepresentations of this $C^{*}$-algebra is equivalent to the given $C^{*}$-category. This generalizes the result of [W2, Theorem 1.3]. Here concrete monoidal $C^{*}$-categories are replaced by abstract monoidal $C^{*}$ categories equipped with monoidal linear ${ }^{*}$-functors.

\section{$\S 1$. The Bialgebra $\operatorname{End}^{\vee}(X)$}

Throughout this section, $\mathcal{C}$ is a monoidal linear category. We denote by $\mathcal{V}_{e c t}$ the monoidal category of finite dimensional vector spaces; here the monoidal product is the usual tensor product, and the monoidal unit is the complex numbers $\mathbf{C}$. We assume that $X: \mathcal{C} \longrightarrow$ Vect $_{f}$ is a monoidal linear functor.

Recall from [JS, §3] that $\operatorname{End}^{\vee}(X)$ is defined as the common coequalizer of the maps

$$
S \longmapsto(S X(\mu), r), \quad S \longmapsto(X(\mu) S, s),
$$

Received by the editors August 7, 1996 and, in revised form, September 23, 1996.

1991 Mathematics Subject Classification. Primary 46L89, 46M15, 18E10.

(C)1998 American Mathematical Society 
from $\operatorname{Hom}(X(s), X(r))$ into $\sum^{\oplus}\{\operatorname{End}(X(r)): r \in O b(\mathcal{C})\}$, for all $\mu \in \operatorname{Hom}(r, s)$. Thus $\operatorname{End}^{\vee}(X)$ is the quotient of $\sum^{\oplus}\{\operatorname{End}(X(r)): r \in O b(\mathcal{C})\}$ by the subspace $\mathcal{V}$ generated by elements of the form

$$
(S X(\mu), r)-(X(\mu) S, s),
$$

for all $S \in \operatorname{Hom}(X(s), X(r))$ and all $\mu \in \operatorname{Hom}(r, s)$. We write $[S]=(S, r)+\mathcal{V}$ for all $S \in \operatorname{End}(X(r))$.

For each object $r$ of $\mathcal{C}$, we pick a basis $\left\{e_{i}^{r}\right\}$ for $X(r)$. Put $e_{i, j}^{r}=e_{i}^{r *} \otimes e_{j}^{r}$. Then $\left\{e_{i, j}^{r}\right\}$ is a basis for $\operatorname{End}(X(r))$. We define a linear map $\gamma_{r}: X(r) \longrightarrow$ $X(r) \otimes \operatorname{End}^{\vee}(X)$ by

$$
\gamma_{r}(u)=\sum_{i, j} e_{i, j}^{r}(u) \otimes\left[e_{j, i}^{r}\right], \quad \forall u \in X(r) .
$$

Then each $\gamma_{r}$ is a comodule structure on $X(r)$, and $\gamma: X \longrightarrow X \otimes \operatorname{End}^{\vee}(X)$ is a monoidal-preserving natural transformation. The vector space $\operatorname{End}^{\vee}(X)$ is a bialgebra with comultiplication $\Delta_{0}$, counit $\epsilon_{0}$ and multiplication given by

$$
\begin{gathered}
\Delta_{0}\left(\left[e_{i, j}^{r}\right]\right)=\sum_{k}\left[e_{i, k}^{r}\right] \otimes\left[e_{k, j}^{r}\right], \\
\epsilon_{0}([S])=\operatorname{Tr}(S), \quad[S][T]=[S \otimes T] .
\end{gathered}
$$

Let $\operatorname{Comod}_{f}\left(\operatorname{End}^{\vee}(X)\right)$ denote the monoidal category of finite dimensional right $\operatorname{End}^{\vee}(X)$-comodules. Using the natural transformation $\gamma$, we obtain a monoidal linear functor

$$
\widehat{X}: \mathcal{C} \longrightarrow \mathcal{C}_{\operatorname{omod}}\left(\operatorname{End}^{\vee}(X)\right),
$$

given by $\widehat{X}(r)=\left(X(r), \gamma_{r}\right)$. We recall the following standard result from Theorem 3 of [JS, §7].

Theorem 1.1. If $\mathcal{C}$ is abelian and $X$ is exact and faithful, then $\widehat{X}$ is an equivalence of categories.

For each object $r$ of $\mathcal{C}$, put

$$
\Gamma_{r}=\sum_{i, j} e_{i, j}^{r} \otimes\left[e_{j, i}^{r}\right], \quad \forall u \in X(r) .
$$

Then $\Gamma_{r}$ is a corepresentation of the bialgebra $\operatorname{End}^{\vee}(X)$, that is,

$$
\left(i d \otimes \Delta_{0}\right)\left(\Gamma_{r}\right)=\left(\Gamma_{r} \otimes I\right)(i d \otimes \tau)\left(\Gamma_{r} \otimes I\right),
$$

where $\tau$ is the twist map $\tau(a \otimes b)=b \otimes a$. Since $\gamma$ is a monoidal-preserving natural transformation, it follows that

$$
\begin{aligned}
\Gamma_{r} \diamond \Gamma_{s} & =\Gamma_{r s}, \\
\Gamma_{s}(X(\mu) \otimes I) & =(X(\mu) \otimes I) \Gamma_{r} .
\end{aligned}
$$

for all $\mu \in \operatorname{Hom}(r, s)$ and all objects $r, s$ in $\mathcal{C}$. Also note that

$$
\begin{gathered}
\gamma_{r}(u)=\Gamma_{r}(u \otimes I), \quad \forall u \in X(r), \\
{[S]=(\operatorname{Tr} \otimes i d)\left(\Gamma_{r}(S \otimes I)\right), \quad \forall S \in \operatorname{End}(X(r)) .}
\end{gathered}
$$


Suppose that $\mathcal{C}$ is left autonomous. We recall from $[\mathrm{JS} 2, \S 7]$ that a monoidal category $\mathcal{C}$ is said to be left autonomous if for any object $r$, there is an object $\tilde{r}$ and there are arrows $\vartheta_{r} \in \operatorname{Hom}(\iota, r \tilde{r})$ and $\bar{\vartheta}_{r} \in \operatorname{Hom}(\tilde{r} r, \iota)$ such that

$$
\begin{aligned}
& \left(I_{r} \diamond \bar{\vartheta}_{r}\right)\left(\vartheta_{r} \diamond I_{r}\right)=I_{r}, \\
& \left(\bar{\vartheta}_{r} \diamond I_{\tilde{r}}\right)\left(I_{\tilde{r}} \diamond \vartheta_{r}\right)=I_{\tilde{r}},
\end{aligned}
$$

where $\diamond$ is the monoidal product, and $\iota$ is the monoidal unit of $\mathcal{C}$. We call $\tilde{r}$ a left dual of $r$, and we refer to the pair $\left(\vartheta_{r}, \bar{\vartheta}_{r}\right)$ as an adjunction between $\tilde{r}$ and $r$. We then deduce that that there is a bijective antilinear map $J_{r}: X(r) \longrightarrow X(\tilde{r})$ such that

$$
\begin{aligned}
X\left(\vartheta_{r}\right)(1) & =\sum_{i} e_{i}^{r} \otimes J_{r} e_{i}^{r}, \\
X\left(\bar{\vartheta}_{r}\right)\left(J_{r} u_{2} \otimes u_{1}\right) & =\left\langle u_{1}, u_{2}^{*}\right\rangle, \quad \forall u_{1}, u_{2} \in X(r),
\end{aligned}
$$

where $u_{2}^{*}$ is the image of $u_{2}$ in the dual space $X(r)^{*}$. For each $S \in \operatorname{End}(X(r))$, put

$$
S^{\sharp}=\left(X\left(\bar{\vartheta}_{r}\right) \otimes I_{\tilde{r}}\right)\left(I_{\tilde{r}} \otimes S \otimes I_{\tilde{r}}\right)\left(I_{\tilde{r}} \otimes X\left(\vartheta_{r}\right)\right) .
$$

Then $\operatorname{End}^{\vee}(X)$ admits an antipode $\nu$ (see [JS, $\left.\S 9\right]$ ) given by $\nu([S])=\left[S^{\sharp}\right]$.

\section{§2. Compact quantum Groups associated With monoidal FunCtors}

Throughout this section, $\mathcal{R}$ is a strict monoidal abelian $C^{*}$-category; we assume that the monoidal unit $\iota$ is irreducible. Note that since $\mathcal{R}$ is abelian, it follows that $\mathcal{R}$ has subobjects and direct sums in the sense of [DR, §1]. We denote by $\mathcal{H i l}_{f}$ the strict monoidal $C^{*}$-category of finite dimensional Hilbert spaces; here the monoidal product is the usual tensor product, and the monoidal unit is the complex numbers C. If $Q$ is a compact quantum group, we denote by $\mathcal{U} \operatorname{corep}_{f}(Q)$ the strict monoidal $C^{*}$-category of finite dimensional unitary corepresentations of $Q$.

Observe that if $\mathcal{R}$ is left autonomous, then it is also right autonomous. If $\left(\vartheta_{r}, \bar{\vartheta}_{r}\right)$ is an adjunction between $\tilde{r}$ and $r$, then $\left(\bar{\vartheta}_{r}^{*}, \vartheta_{r}^{*}\right)$ is an adjunction between $r$ and $\tilde{r}$. We will choose $\vartheta_{\tilde{r}}=\bar{\vartheta}_{r}^{*}$ and $\bar{\vartheta}_{\tilde{r}}=\vartheta_{r}^{*}$.

The category $\mathcal{H i l}_{f}$ is left autonomous in the following way. For each object $V$ of $\mathcal{H i l}_{f}$, let $W$ be an object of $\mathcal{H i l}_{f}$ equipped with a bijective antilinear map $J: V \longrightarrow W$. Pick an orthonormal basis $\left\{e_{i}\right\}$ for $V$, and define

$$
\begin{aligned}
t_{J}(1) & =\sum_{i} e_{i} \otimes J e_{i}, \\
\bar{t}_{J}\left(J v_{2} \otimes v_{1}\right) & =\left\langle v_{1} \mid v_{2}\right\rangle, \quad \forall v_{1}, v_{2} \in V .
\end{aligned}
$$

Then $W$ is a left dual of $V$ with an adjunction $\left(t_{J}, \bar{t}_{J}\right)$. Also $V$ is a left dual of $W$ with an adjunction $\left(\bar{t}_{J}^{*}, t_{J}^{*}\right)$. Note that $\bar{t}_{J}^{*}=t_{J^{-1}}$ and $t_{J}^{*}=\bar{t}_{J^{-1}}$, with respect to an orthonormal basis $\left\{f_{i}\right\}$ of $W$.

If $Q$ is a compact quantum group, then the category $\mathcal{U}$ corep $p_{f}(Q)$ is left autonomous in the following way. For each object $\left(\alpha, V_{\alpha}\right)$ of $\mathcal{U}$ corep $f(Q)$, put $\widetilde{\alpha}=$ $\alpha^{J \otimes *}$, where $J: V_{\alpha} \longrightarrow \tilde{V}_{\alpha}$ is the canonical antilinear map. Since $\alpha$ is unitary, it follows that $t_{J} \in \operatorname{Hom}(\iota, \alpha \diamond \widetilde{\alpha})$ and $\bar{t}_{J} \in \operatorname{Hom}(\widetilde{\alpha} \diamond \alpha, \iota)$. Thus $\left(\widetilde{\alpha}, \tilde{V}_{\alpha}\right)$ is a left dual of $\left(\alpha, V_{\alpha}\right)$ with an adjunction $\left(t_{J}, \bar{t}_{J}\right)$.

Theorem 2.1. Suppose that $\mathcal{R}$ is a left autonomous strict monoidal abelian $C^{*}$ category. Assume that there is an exact faithful monoidal linear ${ }^{*}$-functor $X$ : 
$\mathcal{R} \longrightarrow \mathcal{H i l}_{f}$. Then there is a $C^{*}$-norm on $\operatorname{End}^{\vee}(X)$, and the completion $Q$ of $\operatorname{End}^{\vee}(X)$ under this norm is a compact quantum group.

Let $r$ be any object of $\mathcal{R}$. Since $X: \mathcal{R} \longrightarrow \mathcal{H i l}_{f}$ is faithful, $\operatorname{Hom}(r, r)$ is a finite dimensional $C^{*}$-algebra. Hence there are minimal projections $\phi_{i} \in \operatorname{Hom}(r, r)$ such that $\phi_{i} \phi_{j}=\delta_{i j}$ and $\sum_{i} \phi_{i}=I_{r}$. Since $\mathcal{R}$ has subobjects, there are arrows $\mu_{i} \in \operatorname{Hom}\left(r_{i}, r\right)$ and $\mu_{i}^{*} \in \operatorname{Hom}\left(r, r_{i}\right)$ such that $\mu_{i} \mu_{i}^{*}=\phi_{i}$ and $\mu_{i}^{*} \mu_{i}=I_{r_{i}}$. Since the $\phi_{i}$ are minimal, the $r_{i}$ are irreducible. Since $\phi_{i} \phi_{j}=\delta_{i j}$, we have $\mu_{i}^{*} \mu_{j}=\delta_{i j}$. Therefore $r=\sum_{i}^{\oplus} r_{i}$.

Put $t_{r}=X\left(\vartheta_{r}\right)$ and $\bar{t}_{r}=X\left(\bar{\vartheta}_{r}\right)$. Since $X$ is monoidal, $\left(t_{r}, \bar{t}_{r}\right)$ is an adjunction between $X(\tilde{r})$ and $X(r)$. Let $\tilde{J}: X(r) \longrightarrow \widetilde{X(r)}$ be the canonical antilinear map. Then $\left(\bar{t}_{r} \otimes I\right)\left(I \otimes t_{\tilde{J}}\right)$ is a bijective linear map from $X(\tilde{r})$ onto $\widetilde{X(r)}$, and hence $\operatorname{dim}(X(\tilde{r}))=\operatorname{dim}(\widehat{X(r)})$. Therefore there is a bijective antilinear map $J: X(r) \longrightarrow$ $X(\tilde{r})$ such that $\left\langle J u_{1} \mid J u_{2}\right\rangle=\left\langle u_{2} \mid u_{1}\right\rangle$ for all $u_{1}, u_{2} \in X(r)$. Put $\psi=\left(\bar{t}_{r} \otimes I\right)\left(I \otimes t_{J}\right)$; then it is a bijective linear map on $X(\tilde{r})$. We then deduce that

$$
t_{r}=\left(I \otimes \psi^{-1}\right) t_{J}, \quad \bar{t}_{r}=\bar{t}_{J}(\psi \otimes I) .
$$

Put $J_{r}=\psi^{-1} J$. Then we have

$$
t_{r}=t_{J_{r}}, \quad \bar{t}_{r}=\bar{t}_{J_{r}} .
$$

Put $t_{\tilde{r}}=\bar{t}_{r}^{*}$ and $\bar{t}_{\tilde{r}}=t_{r}^{*}$. Since $X$ is a ${ }^{*}$-functor, we get

$$
t_{\tilde{r}}=X\left(\vartheta_{\tilde{r}}\right), \quad \bar{t}_{\tilde{r}}=X\left(\bar{\vartheta}_{\tilde{r}}\right) .
$$

For each object $r$ of $\mathcal{R}$, we pick an orthonormal basis $\left\{e_{i}^{r}\right\}$ for $X(r)$.

Proposition 2.2. For any $r_{1}, \ldots, r_{n} \in \mathcal{J}(\mathcal{R})$, the set

$$
\left\{\left[e_{i j}^{r_{k}}\right]: i, j=1, \ldots, \operatorname{dim}\left(X\left(r_{k}\right)\right), k=1, \ldots, n\right\}
$$

is linearly independent.

Proof. Let $W$ be the vector space generated by the set

$$
\left\{\left[e_{i j}^{r_{k}}\right]: i, j=1, \ldots, \operatorname{dim}\left(X\left(r_{k}\right)\right), k=1, \ldots, n\right\} .
$$

To prove this proposition, it is sufficient to show that for any finite set of complex numbers

$$
\left\{c_{i j}^{k}: i, j=1, \ldots, \operatorname{dim}\left(X\left(r_{k}\right)\right), k=1, \ldots, n\right\},
$$

there is a linear functional $\rho \in W^{*}$ such that $\rho\left(\left[e_{i j}^{r_{k}}\right]\right)=c_{i j}^{k}$ for all $i, j$ and $k$. Let $B=\sum_{k}^{\oplus} \operatorname{End}\left(X\left(r_{k}\right)\right)$, and put

$$
A=\left\{\sum_{k}^{\oplus}(i d \otimes \rho)\left(\Gamma_{r_{k}}\right): \rho \in W^{*}\right\} .
$$

Then $A$ is a subalgebra of $B$. Since the functor $\widehat{X}$ of Theorem 1.1 is fully faithful, it follows that $\Gamma_{r_{1}}, \ldots, \Gamma_{r_{n}}$ are pairwise nonequivalent irreducible. We then deduce that $X\left(r_{1}\right), \ldots, X\left(r_{n}\right)$ are pairwise nonisomorphic simple $A$-modules. Therefore

$$
A^{\prime}=\operatorname{End}_{A}\left(\sum_{k}^{\oplus} X\left(r_{k}\right)\right)=\sum_{k}^{\oplus} \mathbf{C} I_{r_{k}} .
$$

Thus $B$ is contained in the bicommutant $A^{\prime \prime}$. By the Jacobson density theorem, for any $T=\sum_{k}^{\oplus} T_{k} \in B$, there is an element $a=\sum_{k}^{\oplus}(i d \otimes \rho)\left(\Gamma_{r_{k}}\right) \in A$ such that 
$a e_{i}^{r_{k}}=T e_{i}^{r_{k}}$ for all $i$ and $k$. For each $k=1, \ldots, n$, if we take $T_{k}=\sum_{i j} c_{j i}^{k} e_{i j}^{r_{k}}$, then we get

$$
\sum_{i j} \rho\left(\left[e_{j i}^{r_{k}}\right]\right) e_{i j}^{r_{k}}=\sum_{i j} c_{j i}^{k} e_{i j}^{r_{k}}
$$

Proposition 2.3. Put $Q_{0}=\sum_{r \in \mathcal{J}(\mathcal{R})}^{\oplus}[\operatorname{End}(X(r))]$. Then $\operatorname{End}^{\vee}(X)=Q_{0}$.

Proof. Let $S \in \operatorname{End}(X(s))$. Choose a decomposition $I_{s}=\sum_{i} \mu_{i} \mu_{i}^{*}$ with $\mu_{i} \in$ $\operatorname{Hom}\left(s_{i}, s\right)$ and $s_{i} \in \mathcal{J}(\mathcal{R})$. Then we have

$$
[S]=\sum_{i}\left[X\left(\mu_{i}^{*}\right) S X\left(\mu_{i}\right)\right]
$$

Therefore $\operatorname{End}^{\vee}(X)$ is generated by

$$
\{[R]: R \in \operatorname{End}(X(r)), r \in \mathcal{J}(\mathcal{R})\} .
$$

We deduce from Proposition 2.2 that

$$
\left\{\left[e_{i j}^{r}\right]: i, j=1, \ldots, \operatorname{dim}(X(r)), r \in \mathcal{J}(\mathcal{R})\right\}
$$

is a basis for $\operatorname{End}^{\vee}(X)$, and this proves the proposition.

We define an involution operation * on $\operatorname{End}^{\vee}(X)$ by

$$
[S]^{*}=\left[S^{* \sharp}\right]=[\tilde{S}] .
$$

Then $\operatorname{End}^{\vee}(X)$ becomes a unital *-algebra.

For each object $r$ of $\mathcal{R}$, we have

$$
\widetilde{\Gamma}_{r}=\sum_{i j} \widetilde{e_{i j}^{r}} \otimes\left[\widetilde{e_{j i}^{r}}\right]=\sum_{i j} e_{i j}^{\tilde{r}} \otimes\left[e_{j i}^{\tilde{r}}\right]=\Gamma_{\tilde{r}} .
$$

Since $\vartheta_{r} \in \operatorname{Hom}(\iota, r \tilde{r})$ and $\bar{\vartheta}_{r} \in \operatorname{Hom}(\tilde{r} r, \iota)$, it follows that

$$
\begin{aligned}
& \left(\Gamma_{r} \diamond \widetilde{\Gamma}_{r}\right)\left(t_{r} \otimes I\right)=t_{r} \otimes I, \\
& \left(\bar{t}_{r} \otimes I\right)\left(\widetilde{\Gamma}_{r} \diamond \Gamma_{r}\right)=\bar{t}_{r} \otimes I .
\end{aligned}
$$

Therefore $\Gamma_{r}$ is a unitary.

We define a linear functional $h_{0}$ on $Q_{0}$ by

$$
h_{0}\left(\left[S_{r}\right]\right)= \begin{cases}1, & \text { if }\left[S_{r}\right]=[1] \\ 0, & \text { if } S_{r} \in \operatorname{End}(X(r)), r \neq \iota .\end{cases}
$$

Proposition 2.4. Let $r, s \in \mathcal{J}(\mathcal{R})$ and $r \neq s$.

(i) For any $S_{r} \in \operatorname{End}(X(r))$ and $S_{s} \in \operatorname{End}(X(s))$,

$$
h_{0}\left(\left[S_{s}\right]^{*}\left[S_{r}\right]\right)=0 .
$$

(ii) For any $S_{r}, T_{r} \in \operatorname{End}(X(r))$,

$$
h_{0}\left(\left[T_{r}\right]^{*}\left[S_{r}\right]\right)=\operatorname{dim}(X(r))^{-1} \sum_{m}\left\langle S_{r} e_{m}^{r} \mid T_{r} e_{m}^{r}\right\rangle .
$$

(iii) For any nonzero $a=\sum_{r}^{\oplus}\left[S_{r}\right] \in Q_{0}$,

$$
h_{0}\left(a^{*} a\right)=\sum_{r} \operatorname{dim}(X(r))^{-1} \sum_{m}\left\|S_{r} e_{m}^{r}\right\|^{2}>0 .
$$


Proof. (i) Choose a decomposition $I_{\tilde{r} s}=\sum_{i} \mu_{i} \mu_{i}^{*}$ with $\mu_{i} \in \operatorname{Hom}\left(r_{i}, \tilde{r} s\right)$ and $r_{i} \in \mathcal{J}(\mathcal{R})$. We then have

$$
\begin{aligned}
h_{0}\left(\left[S_{s}\right]^{*}\left[S_{r}\right]\right) & =h_{0}\left(\left[\tilde{S}_{s} \otimes S_{r}\right]\right) \\
& =\sum_{r_{i}=\iota} h_{0}\left(\left[X\left(\mu_{i}^{*}\right)\left(\tilde{S}_{s} \otimes S_{r}\right) X\left(\mu_{i}\right)\right]\right) .
\end{aligned}
$$

Note that for any objects $r, s$ and $t, \mu \longmapsto\left(I_{\tilde{r}} \diamond \mu\right)\left(\vartheta_{\tilde{r}} \diamond I_{t}\right)$ is a bijective linear map from $\operatorname{Hom}(r t, s)$ onto $\operatorname{Hom}(t, \tilde{r} s)$. Since $\operatorname{Hom}(r, s)=\{0\}$, it follows that $X\left(\mu_{i}\right)=0$ for all $i$ with $r_{i}=\iota$. This proves (i).

(ii) By similar arguments as in (i) with $r=s$, we get

$$
h_{0}\left(\left[T_{r}\right]^{*}\left[S_{r}\right]\right)=\sum_{r_{i}=\iota} h_{0}\left(\left[X\left(\mu_{i}^{*}\right)\left(\tilde{T}_{s} \otimes S_{r}\right) X\left(\mu_{i}\right)\right]\right) .
$$

Since $\operatorname{Hom}(\iota, \tilde{r} r)=\mathbf{C} \bar{\vartheta}_{r}^{*}$ and $\operatorname{Hom}(\tilde{r} r, \iota)=\mathbf{C} \bar{\vartheta}_{r}$, it follows that

$$
\sum_{r_{i}=\iota} X\left(\mu_{i}\right) X\left(\mu_{i}^{*}\right)=c X\left(\bar{\vartheta}_{r}^{*}\right) X\left(\bar{\vartheta}_{r}\right),
$$

for some scalar $c$. For any $i$ with $r_{i} \neq \iota$, we have $\mu_{i}^{*} \bar{\vartheta}_{r}^{*} \in \operatorname{Hom}\left(\iota, r_{i}\right)=\{0\}$. Hence

$$
\begin{aligned}
\bar{t}_{r}^{*} & =X\left(\bar{\vartheta}_{r}^{*}\right)=X\left(\sum_{i} \mu_{i} \mu_{i}^{*} \bar{\vartheta}_{r}^{*}\right)=X\left(\sum_{r_{i}=\iota} \mu_{i} \mu_{i}^{*} \bar{\vartheta}_{r}^{*}\right) \\
& =c X\left(\bar{\vartheta}_{r}^{*}\right) X\left(\bar{\vartheta}_{r}\right) X\left(\bar{\vartheta}_{r}^{*}\right)=c \bar{t}_{r}^{*} \bar{t}_{r} \bar{t}_{r}^{*} .
\end{aligned}
$$

Thus $c \bar{t}_{r} \bar{t}_{r}^{*}(1)=1$. Observe that

$$
\begin{gathered}
\bar{t}_{r} \bar{t}_{\tilde{r}}^{*}(1)=\sum_{i}\left\langle J_{r}^{-1} e_{i}^{\tilde{r}} \mid J_{r}^{-1} e_{i}^{\tilde{r}}\right\rangle=\operatorname{dim}(X(r)), \\
\bar{t}_{r}\left(\tilde{T}_{r} \otimes S_{r}\right) \bar{t}_{r}^{*}(1)=\sum_{m}\left\langle S_{r} e_{m}^{r} \mid T_{r} e_{m}^{r}\right\rangle .
\end{gathered}
$$

Now we have

$$
\begin{aligned}
h_{0}\left(\left[T_{r}\right]^{*}\left[S_{r}\right]\right) & =h_{0}\left(\left[\sum_{r_{i}=\iota} X\left(\mu_{i}\right) X\left(\mu_{i}^{*}\right)\left(\tilde{T}_{s} \otimes S_{r}\right)\right]\right) \\
& =c h_{0}\left(\left[X\left(\bar{\vartheta}_{r}^{*}\right) X\left(\bar{\vartheta}_{r}\right)\left(\tilde{T}_{s} \otimes S_{r}\right)\right]\right) \\
& =c h_{0}\left(\left[\bar{t}_{r}\left(\tilde{T}_{s} \otimes S_{r}\right) \bar{t}_{r}^{*}\right]\right) \\
& =c h_{0}\left(\left[\sum_{m}\left\langle S_{r} e_{m}^{r} \mid T_{r} e_{m}^{r}\right\rangle 1\right]\right) \\
& =\operatorname{dim}(X(r))^{-1} \sum_{m}\left\langle S_{r} e_{m}^{r} \mid T_{r} e_{m}^{r}\right\rangle .
\end{aligned}
$$

(iii) It is a consequence of (i) and (ii).

Proof of Theorem 2.1. For each $a \in Q_{0}$, we put

$$
\begin{aligned}
\|a\|=\sup \{\|\pi(a)\|: & \pi \text { is a nondegenerate } \\
& \text { representation on Hilbert spaces }\} .
\end{aligned}
$$


Since each $\Gamma_{r}$ is unitary, $\|a\|$ is finite and hence $\|\cdot\|$ is a $C^{*}$-seminorm on $Q_{0}$. The ideal $\mathcal{I}_{0}$ of $Q_{0}$ consisting of elements of seminorm zero is closed under the involution operation *. The canonical quotient map $q: Q_{0} \longrightarrow Q_{0} / \mathcal{I}_{0}$ is a unital *-homomorphism. Let $Q$ denote the completion of $Q_{0} / \mathcal{I}_{0}$. We represent the $C^{*}$ algebra $Q$ on a Hilbert space by a faithful nondegenerate representation, and then see that

$$
\left\|(q \otimes q) \circ \Delta_{0}(a)\right\| \leq\|a\|, \quad \forall a \in Q_{0} .
$$

We then deduce that there is a comultiplication $\Delta: Q \longrightarrow Q \otimes Q$ such that

$$
\Delta(q(a))=(q \otimes q) \circ \Delta_{0}(a), \quad \forall a \in Q_{0} .
$$

Each $\beta_{r}=(i d \otimes q)\left(\Gamma_{r}\right)$ is a unitary corepresentation of $(Q, \Delta)$, and the matrix elements of the family $\left\{\beta_{r}\right\}$ generate $Q$. By Remark 2.6(b) of [B], there exists a unique Haar measure $h$ for $\left(Q, \Delta,\left\{\beta_{r}\right\}\right)$. Let $r \in \mathcal{J}(\mathcal{R})$ with $r \neq \iota$. Since the unit $I$ of $Q_{0}$ is not in $\mathcal{I}_{0}$, it follows that $q(I)$ is not in the linear span of $q\left(\left[e_{i j}^{r}\right]\right)$ for all $i, j$. By the Hahn-Banach theorem, we can choose $\eta \in Q^{*}$ such that $\eta(q(I))=1$ and $\eta\left(q\left(\left[e_{i j}^{r}\right]\right)\right)=0$ for all $i, j$. Using Theorem 2.3 of $[\mathrm{B}]$, we get

$$
\begin{aligned}
h\left(q\left(\left[e_{i j}^{r}\right]\right)\right) & =(\eta * h)\left(q\left(\left[e_{i j}^{r}\right]\right)\right) \\
& =\sum_{k} \eta\left(q\left(\left[e_{i k}^{r}\right]\right)\right) h\left(q\left(\left[e_{k j}^{r}\right]\right)\right)=0 .
\end{aligned}
$$

Therefore $h \circ q=h_{0}$. If $\pi$ is the cyclic representation of $Q$ induced by the positive linear functional $h$, then it follows Proposition 2.4(iii) that $\pi \circ q$ is a faithful nondegenerate representation of $Q_{0}$. Therefore $\mathcal{I}_{0}=\{0\}$ and $\|\cdot\|$ is a $C^{*}$-norm.

The following theorem contains Theorem 1.3 of [W2] as a special case.

Theorem 2.5. The functor $\Pi: \mathcal{R} \longrightarrow \mathcal{U}_{\text {corep }_{f}}(Q)$, given by $\Pi(r)=\Gamma_{r}$ and $\Pi(\mu)=X(\mu)$, is an equivalence of monoidal categories.

Proof. Since $\widehat{X}$ is fully faithful, so is $\Pi$. To prove $\Pi$ is an equivalence, let $\alpha$ be any object of $\mathcal{U} \operatorname{corep}_{f}(Q)$. We need to prove that $\alpha$ is equivalent to $\Pi(r)$ for some $r$ in $\mathcal{R}$. By Theorem 2.4 of [B], $\alpha$ is the direct sum of irreducible unitary subcorepresentations $\alpha_{i}$. The family $\mathcal{L}=\left\{\Gamma_{r}: r \in \mathcal{J}(\mathcal{R})\right\}$ consists of mutually nonequivalent irreducible corepresentations of $Q$, and the matrix elements of $\mathcal{L}$ generate $Q_{0}$. By Theorem 2.5 of [B], each $\alpha_{i}$ is equivalent to an element $\Gamma_{r_{i}}$ of $\mathcal{L}$. If we put $r=\sum^{\oplus} r_{i}$, then $\Pi(r)=\Gamma_{r}$ is equivalent to $\sum^{\oplus} \Gamma_{r_{i}}$. Hence $\alpha$ is equivalent to $\Pi(r)$.

\section{ACKNOWLEDGEMENT}

This research was supproted by an ARC Small Grant at Macquarie University. The author would like to thank Professor R. Street for his support.

\section{REFERENCES}

[B] H. H. Bui, Compact quantum groups and their corepresentations, Bull. Austral. Math. Soc., to appear.

[B2] H. H. Bui, Morita equivalence of twisted crossed products by coactions, J. Funct. Anal. 123 (1994), 59-98. MR 95g:46121

[BS] S. Baaj and G. Skandalis, Unitaires multiplicatifs et dualite pour les produits crossés de $C^{*}$-algèbres, Ann. Sci. Ec. Norm. Sup. 26 (1993), 425-488. MR 94e:46127 
[D] P. Deligne, Catégories Tannakiennes, The Grothendieck Festschrift, Progress in Math. 87, Birkhäuser, 1990, pp. 111-195. MR 92d:14002

[DR] S. Doplicher and J. E. Roberts, A new duality theory for compact groups, Invent. Math. 98 (1989), 157-218. MR 90k:22005

[JS] A. Joyal and R. Street, An introduction to Tannaka duality and quantum groups, Lecture Notes in Mathematics, Vol. 1488, Springer-Verlag, New York, 1991, pp. 413-492. MR 93f: 18015

[JS2] A. Joyal and R. Street, Braided tensor categories, Adv. Math. 102 (1993), 20-78. MR 94m: 18008

[K] C. Kassel, Quantum groups, Graduate Texts in Mathematics, Vol. 155, Springer-Verlag, New York, 1995. MR 96e: 17041

[KT] C. Kassel and V. Turaev, Double construction for monoidal categories, Acta Math. 175 (1995), 1-48. MR 96m:18015

[Sc] P. Schauenburg, Tannaka duality for arbitrary Hopf algebras, Algebra-Berichte Vol. 66, Verlag Reinhard Fisher Munchen, 1992.

[Sk] G. Skandalis, Operator algebras and Duality,, Proceedings of the International Congress of Mathematicians, vol. 2, 1990, pp. 997-1009. MR 93d:46094

[W] S. L. Woronowicz, Compact matrix pseudogroups, Commun. Math. Phys. 111 (1987), 613665. MR 88m:46079

[W2] S. L. Woronowicz, Tannaka-Krein duality for compact matrix pseudogroups. Twisted SU(N) groups, Invent. Math. 93 (1988), 35-76. MR 90e:22033

[Y] D. N. Yetter, Quantum groups and representations of monoidal categories, Math. Proc. Cambridge Philos. Soc. 108 (1990), 261-290. MR 91k:16028

Department of Mathematics, Macquarie University, New South Wales 2109, AusTRALIA

Current address: School of Mathematics, The University of New South Wales, Sydney, New South Wales 2052, Australia

E-mail address: hung@alpha.maths.unsw.edu.au 